HELLMUT PUSCHMANN*

\title{
ES MUSS ZUSAMMENKOMMEN, WAS ZUSAMMENGEHÖRT
}

\section{Caritasarbeit im real existierenden Sozialismus in der ehemaligen DDR}

(Erhalten: 22 November 2007; angenommen: 13 Februar 2008)

In der ehemaligen DDR konnte die Katholische Kirche eine breite Caritasarbeit aufbauen, die weitgehend die Ressourcen der Gemeinden nutzte und damit eine enge Verbindung zu den Pfarrgemeinden hatte. Es waren nicht nur Hilfen auf Gemeindebasis möglich, sondern auch durch eine Vielzahl von Einrichtungen, wie Altenpflegeheime, Krankenhäuser, Heime für geistig Behinderte, Kindergärten und Erholungsheime. Diese wurden auch gern von Menschen angenommen, die nicht zur Kirche gehörten. Diese Arbeit konnte die Kirche eigenverantwortlich leisten ohne staatliche Steuerung. Viele finanzielle und fachliche Hilfen von der Deutschen Bischofskonferenz, dem Deutschen Caritasverband, dem Bonifatiuswerk der deutschen Katholiken und einzelnen Personen haben dieses Wirken entscheidend unterstützt und ermöglicht. Als nach der Wiedererlangung der Einheit Deutschlands die Caritas der einzelnen ostdeutschen Jurisdiktionsbezirke wieder formell Mitglied im Deutschen Caritasverband werden konnten, vollendete sich ein langer gemeinsamer Weg, der durch die Politik schwierig, aber nicht unmöglich gemacht wurde.

Schlüsselbegriffe: Diakonie, DDR, Deutschland, Katholische Kirche, Kommunismus, Staatssozialismus, Diktatur, Geschichte, Rückblick, helfender Beruf

Parts That Belong Together Are to Join: Charitable Work in the Real Socialism of the Former GDR: In the former GDR the Catholic Church was able to establish comprehensive charitable activities that to a large extent were based on the resources of the faithful, and therefore had close ties with parish communities. Help could be extended not only at the level of church communities, but also through numerous institutions like homes for the elderly, hospitals, and homes and resorts caring for people with mental disabilities. These were also gladly visited by people who did not belong to the church. The church was allowed to carry out this work

* Hellmut Puschmann, Caritasverband für das Bistum Dresden-Meißen e. V., Magdeburger Str. 33, D-01067 Dresden; Hellmut.Puschmann@t-online.de.

1788-4934 / \$20.00 (C) 2008 Akadémiai Kiadó, Budapest 
independently, without direct state control. Financial and professional support from the German Catholic Bishops' Conference, the German Caritas Association, the Bonifatiuswerk organisation of German Catholics and certain private individuals were decisive in enabling these activities. When following the reunification of Germany the charitable organisation responsible for the individual East German public administration regions could again become an official member of the German Caritas Association, progress along a common path that was hindered but never prevented by politics could to continue.

Keywords: diaconate, GDR, Germany, Catholic Church, communism, state socialism, dictatorship, history, retrospect, helping profession

\section{Einleitung}

Im Frühsommer 1990 wurden die diözesanen Caritasstellen ${ }^{1}$ in Ostdeutschland entsprechend den neuen Möglichkeiten wieder in die Rechtsform als eingetragener Verein überführt. Als im Herbst 1990 die Diözesancaritasverbände aus den neuen Bundesländern wieder in den Deutschen Caritasverband aufgenommen wurden, wurden die Strukturen der deutschen Caritas wieder in ihre Normalform gebracht, die seit der Trennung Deutschlands verwehrt war. Dies war ein Schritt, der im Unterschied zur Jahrzehnte langen staatlich-politischen Distanzierung beider deutscher Staaten voneinander „nur“ juristisch vollzogen werden musste. Die Kirche und auch die Caritas hatten immer Formen gefunden, ihre Gemeinschaft zu leben. Auf dem Gebiet der DDR allein gab es kirchenrechtlich nur eine wirkliche Diözese, das Bistum Meißen (seit 1980 Dresden-Meißen). Die Diözese Berlin umfasste sowohl Westberlin als auch Ostberlin und weite Teile im Norden der DDR. Weitere Bereiche gehörten zu Diözesen in Westdeutschland und waren von der politischen Teilung sehr betroffen: Thüringen mit dem kirchlichen Schwerpunkt Erfurt und dem Eichsfeld gehörte zu Fulda, Sachsen-Anhalt mit Magdeburg zum Erzbistum Paderborn und Mecklenburg mit Schwerin zur Diözese Osnabrück. Der Vatikan hat bis zuletzt eine kirchliche Trennung vermeiden können, nutzte jedoch die kirchenrechtlichen Möglichkeiten, um durch die Einsetzung Apostolischer Administratoren in Erfurt, Magdeburg und Schwerin eigenständiges pastorales Handeln zu ermöglichen. Görlitz war ein Sonderfall, weil es der Restteil des ehemaligen Erzbistums Breslau in der DDR war. Dort wurde eine Apostolische Administratur errichtet. Mit diesen Strukturen war es auch möglich, diese sechs Jurisdiktionsbezirke in einer Bischofskonferenz zusammenzufassen, ohne dass dies eine nationale Bischofskonferenz war, was die Anerkennung der Spaltung Deutschlands durch die Katholische Kirche bedeutet hätte.

\footnotetext{
${ }^{1}$ Der Autor des Artikels ist seit 37 Jahren in leitenden Positionen der Caritas tätig. Von 1982 bis 1991 war er Leiter der Zentralstelle Berlin des Deutschen Caritasverbandes in der ehemaligen DDR, dann - als eine der ersten Persönlichkeiten aus Ostdeutschland in ein Amt mit bundesweiter Verantwortung gewählt - 12 Jahre lang Präsident des Deutschen Caritasverbandes Freiburg. Seit 2003 ist er Vorsitzender des Diözesancaritasverbandes Dresden-Meißen und Diözesanpräses des Kolpingwerkes (Angaben der Redaktion).
} 
Die innere Zusammengehörigkeit zwischen dem Deutschen Caritasverband und der Caritas in den Jurisdiktionsgebieten der DDR war entsprechend der inneren Einheit der Kirche stets gewahrt worden, auch wenn äußere Zwänge unterschiedliche Lebensäußerungen dieser einen Wirklichkeit erforderten ${ }^{2}$. Viele Hilfen materieller, finanzieller Art und vor allem durch die Weitergabe von Know-how bei Besuchen westdeutscher Experten halfen, sich kompetent den Aufgaben zu stellen. In der DDR gab es keine Wohlfahrtsverbände im Sinne freier bürgerschaftlicher Initiativen. Der Staat organisierte das gesamte gesellschaftliche Leben. Nur den Kirchen hatte man 1945 nicht verboten, im Rahmen kirchlichen Lebens auch die Nächstenliebe zu organisieren. So überlebten in der Evangelischen Kirche die „Innere Mission“ und in der Katholischen Kirche die „Caritas“, jedoch als eng an die Kirche angeschlossene und unter ihrem Schutz stehende Bereiche kirchlichen Lebens. Dem Namen nach existierte auch das „Deutsche Rote Kreuz“, es war aber eher eine halbstaatliche Rettungsorganisation, kein Verband im freiheitlichen Sinne.

\section{Soziale Arbeit als Lebensäußerung der Kirche}

Die beiden kirchlichen Organisationen arbeiteten jeweils eigenständig, koordinierten jedoch manche Aktivität miteinander und informierten sich gegenseitig in einem engen Vertrauensverhältnis. Die gemeinsame Not schweißte zusammen. So konnten Mitarbeiter der Caritas bei der Diakonie die Ausbildung zum Heilerziehungspfleger oder eine kirchliche Verwaltungsausbildung machen oder Mitarbeiterinnen der Diakonie bei der Caritas die Ausbildung zur Jugendleiterin, weil diese Ausbildungswege jeweils nur bei einer der beiden Organisationen bestanden. Weiter wurden Strategien und Ergebnisse von vielen Verhandlungen mit staatlichen Stellen ausgetauscht und beraten, wenn dies sinnvoll war.

Damit gab es in der DDR einen einzigartigen kirchenpolitischen Sonderweg im gesamten Ostblock. Die Grundlage dafür legte die sowjetische Besatzungsmacht, indem sie den Kampf der Kirchen gegen das Naziregime honorierte und im furchtbaren Nachkriegselend der vielen ausgebombten, heimatlosen und hungernden Menschen alle denkbaren, also auch kirchliche Hilfen akzeptierte, um die Menschen mit Nahrung, Heizmaterial und Wohnmöglichkeiten zu versorgen. Daher genehmigte man schon damals Hilfslieferungen aus anderen Ländern in die Sowjetische Besatzungszone. Insgesamt kamen bis 1950 etwa 4 Millionen Heimatvertriebene in die DDR. Daraus leitete sich der wichtigste politische Grundsatz ab: Die Hilfe der Kirche wird dann angenommen, wenn der Staat nicht in der Lage ist, seinen allumfassenden Anspruch zu realisieren. Aber die Kirche darf nicht so stark werden, dass sie die Gesellschaft spürbar beeinflusst. Auf dieser Basis wurden die meisten im Kriege zweckentfremdeten Caritasheime zurückgegeben. Unter den ausgesiedelten Menschen befanden

\footnotetext{
${ }^{2}$ Weiterführende Literatur dazu: GRANDE \& TAMMER (1990); GRÜTZ (2004); HÖLLEN (1994); JUNG (2003); KÖSTERS (2001a,b); KÖSTERS \& TisCHNER (2005); KROLL (1998); LANGE (1992); LANGE \& PRUSS (1996); PILVOUSEK (1994); SCHÄFER (1998).
} 
sich oft ganze Altenheime und Kinderheime, die umgehend eine Unterkunft benötigten. Auf diese Weise erhielt die Caritas manches Schloss und manches leer stehende Hotel. ${ }^{3}$ Ordensschwestern haben mit äußerstem Einsatz diese Einrichtungen notdürftig hergerichtet. In einem Altenheim im Bistum Meißen waren im Winter täglich 70 Kohleöfen zu heizen. Es gab keinen Aufzug, die Kohle war schlecht. Es dauerte etwa 15 Jahre, bis die ersten größeren Sanierungen dieser Heime möglich wurden (Anhang).

Der Schwerpunkt in der Tätigkeit der Heime waren alte und pflegebedürftige Menschen. Deren Zahl war groß, viele hatten nach der Umsiedlung nie wieder richtig Fuß gefasst. Die Katholische Kirche hatte jedoch auch Krankenhäuser, Einrichtungen für geistig Behinderte und Erholungsheime. Und es gab kirchliche Kindergärten und Kinderheime! Diese waren ein wachsendes Ärgernis für einen Staat, der seine Politik auf die Erziehung eines neuen Menschentyps ausgerichtet hatte und das Bildungsmonopol beanspruchte. Je stärker die staatliche Politik sich auf diesen ideologischen Schwerpunkt ausrichtete, desto größere Schwierigkeiten hatten die Kinderheime und Kindergärten. Schulräte verweigerten die Umschulungsgenehmigung an den Ort, an dem ein Kinderheim war. Das Aufenthaltsbestimmungsrecht der Eltern wurde in solchen Fällen oft nicht respektiert. Die Heimbibliotheken der Kinderheime wurden kontrolliert und Bücher beschlagnahmt. Die Erziehungsmethoden und -ziele der kirchlichen Heimerziehung wurden bösartig hinterfragt und in den Medien zynisch beschrieben. Dies alles führte sowohl Ende der 50er Jahre als auch Mitte der 60er Jahre dazu, dass etliche Kinderheime nicht mehr ausgelastet waren und umprofiliert werden mussten. Die Kindergärten sollten Auflagen für ihre inhaltliche Arbeit erhalten, die nur unter großen Mühen abgewehrt werden konnten. An eine Erweiterung oder gar einen Neubau von Kindergärten war nicht zu denken. Die Arbeit der Kinderheime und der Kindergärten wurde allein aus kirchlichen Mitteln und durch die sehr hohen Elternbeiträge finanziert. Staatliche Einrichtungen boten ihre Leistung kostenlos an. Hingegen gab es kostendeckende staatliche Pflegesätze seit 1967 für Krankenhäuser, Altenund Pflegeheime und für Einrichtungen für geistig Behinderte. Alle anderen Dienste und Verwaltungsbereiche, die Ausbildungsstätten für kirchliche Erzieherinnen, für Altenhelferinnen und Sozialarbeiter konnten nur existieren, weil sie kirchlich finanziert wurden - entscheidend ermöglicht durch die Hilfe aus den westdeutschen Diözesen und dem Deutschen Caritasverband. Die Basis der Caritas lag in den katholischen Pfarrgemeinden und Dekanaten. Die meisten Gemeinden hatten einen Caritashelferkreis oder eine Elisabethkonferenz und organisierten durch diese gemeinsam mit dem Seelsorger die Hilfen. Immer waren zwar zu wenige Helfer und Helferinnen vorhanden, dennoch bewährt sich dieses System seit Jahrhunderten. Neue Versuche, die durch die Pastoralsynode in Dresden Anfang der 70er Jahre angeregt wurden, etwa durch eine „Sachgruppe Diakonie“, die eine Koordinierung der Caritas der Gemeinde zur

\footnotetext{
${ }^{3}$ Im Bistum Dresden-Meißen erhielt die Caritas beispielsweise die Schlösser Lichtenstein, Purschenstein (später getauscht gegen ein Hotel in Olbernhau), Thammenhain, das Herrenhaus Maxen, Seelingstädt. Die meisten dieser Häuser wurden nach 1990 den ursprünglichen Besitzern wieder zurückgegeben (eigene Erinnerung). Heute sind stattdessen Neubauten an anderen Orten errichtet worden.
} 
Aufgabe hatte, konnten nur in größeren Gemeinden sinnvoll umgesetzt werden. Dabei ist zu berücksichtigen, dass der Bevölkerungsanteil der Katholiken stets bei etwa $3-5 \% \mathrm{lag}^{4}$. Die große Zahl der Heimatvertriebenen führte zwar für einige Jahre zu einer Verdoppelung, der Anteil sank aber durch Weiterwanderung und Tod bis zum Mauerbau stetig. Die Zahl der Pfarreien lag vor 1945 bei etwa 600, im Jahr 1948 bei knapp 1000. Diese Zahl sank dann etwas bis zum Ende der DDR. Von diesen Entwicklungen ist auch der caritative Einsatz in den Gemeinden bestimmt: Die Zahl und die Not bestimmen die Notwendigkeiten der Hilfe und die Möglichkeit der Rekrutierung von Helfern. Auf diese Weise konnte eine christlich motivierte Solidarität gelebt werden, die sich von der staatlich normierten Solidarität sehr unterschied: Es ging nicht um politische Inhalte, nicht um eine Selbstdarstellung, sondern um die Not und die Probleme einzelner Menschen und um das Zeugnis der Liebe Christi.

In den einzelnen Dekanaten bot die Caritas Einzelfallhilfe durch Beratung und Vermittlung an. Dies war jedoch ein Minimalangebot, weil meist nur ein Sozialarbeiter oder eine Sozialarbeiterin finanziert werden konnte. Ein ausdrücklicher Teil ihres Dienstauftrages war die Anleitung und Begleitung der Helfergruppen in den Gemeinden ihres Dekanates. Elementare Hilfen für die Heimatvertriebenen wurden später abgelöst durch Hilfen für alte Menschen (Kohle tragen, einkaufen, Behördengänge erledigen) oder für Haftentlassene (Hilfe bei der Einrichtung ihres Zimmers). In manchen Gemeinden waren auch kirchliche Altenpflegerinnen tätig, deren Arbeit jedoch nur möglich war, wenn die Gemeinde die Finanzierung aufbringen konnte. Schließlich hatte jeder Bischof eine diözesane Caritasstelle mit einem Diözesancaritasdirektor eingerichtet. Diese leiteten und koordinierten die Arbeit, nahmen Teile der Aufsicht über die Caritas wahr und führten in seinem Auftrag Verhandlungen mit den staatlichen Stellen. Auf Bistumsebene wurden in Zusammenarbeit mit der Pastoral auch pastorale und soziale Aktivitäten für Blinde, Gehörlose und geistig Behinderte organisiert.

In Berlin wurde die Gesamtarbeit der Caritas Ostdeutschlands koordiniert, zentrale Verhandlungen geführt, der Kontakt zum Deutschen Caritasverband organisiert, die zentralen Fragen der Ausbildung für caritative Berufe, die gemeinsame Weiterbildung aller Caritasstellen konzipiert und durchgeführt und alle Fragen, die mit Hilfen aus Westdeutschland zusammenhingen, bearbeitet. Damit hatte der Deutsche Caritasverband seine Hauptvertretung in Westberlin beauftragt. Diese arbeitete eng mit dem Büro in Ostberlin zusammen. Daneben konnte durch die beiden Dienststellen vielen Menschen durch Beratung und Vermittlung geholfen werden, wenn Probleme der Übersiedlung nach Westdeutschland auftraten oder Angehörige nach einem Fluchtversuch inhaftiert wurden.

\footnotetext{
${ }^{4}$ Die Katholikenzahlen sind sehr ungenau, da seit 1964 keine Volkszählung mit der Frage nach Religionszugehörigkeit stattfand und insgesamt keine Zusammenarbeit mit den staatlichen Meldestellen möglich war. 1985 wurde mit etwa 1,1 Millionen Katholiken unter ca. 16,6 Millionen Einwohnern gerechnet. Später wurde jedoch deutlich, dass diese Zahl zu hoch geschätzt war. In den Satellitenstädten war die Katholikenzahl am niedrigsten, während im Eichsfeld und in den Dörfern mit sorbischer Bevölkerung in der Oberlausitz eine starke katholische Mehrheit existierte.
} 


\section{Begrenzung durch die Staatsideologie - der Geist des Handelns in der Lücke}

Die Politik der DDR-Regierung gegenüber den Kirchen hatte das Ziel, ihren Einfluss zurückzudrängen, sie als Feinde des Sozialismus und des Friedens zu bezeichnen und damit dem Klassenfeind zuzuordnen. In den letzten Jahren der DDR wurden diese Argumentationen verfeinert und indirekter eingesetzt, weil man international als demokratischer Staat die Reputation nicht verlieren wollte. ${ }^{5}$ Dank der inneren Überzeugung und des Glaubens der Mitarbeiter der Caritas und dank der klaren Richtlinien der Bischöfe für das Verhalten von Mitarbeitern gegenüber dem Staat und dem Ministerium für Staatssicherheit gab es nur ganz wenige Fälle, in denen die kirchliche Geschlossenheit durchbrochen wurde. Dieses Vertrauen zueinander ließ auch ein ganz spezielles Klima und eine tiefe Verbundenheit der Mitarbeiterinnen und Mitarbeiter wachsen. So positiv dies auch während der DDR-Zeit war, so hemmend wirkte es sich nach der Wende aus, fehlte doch bei vielen die Einsicht, nun intensiv über den Bereich einer Diasporakirche hinaus zu denken und die gesamte Gesellschaft mit ihren Problemen als Aufgabe anzunehmen.

Die politischen Rahmenbedingungen in der DDR ließen nicht zu, dass die Caritas eine unabhängige Mitverantwortung für die soziale Gestaltung der Gesellschaft übernahm. Somit konzentrierte sich die Arbeit weitgehend auf den einzelnen Menschen. Dabei setzte man auf die Hoffnung, dass dies auch Auswirkungen auf andere hat:

Krankenpflege:

Die gute und hingebungsvolle Pflege in den kirchlichen Einrichtungen wurde indirekt maßstabsbildend für die Lehrpläne an staatlichen Ausbildungseinrichtungen. Die Ausbildung von Krankenschwestern und -pflegern in kirchlichen Krankenhäusern erfolgte auf der Basis von Vereinbarungen mit dem Staat. Als 1973 eine Umstellung der Ausbildung insgesamt erfolgte, versuchte man zunächst staatlicherseits, der Kirche die Möglichkeit zur Ausbildung künftig zu nehmen. In den darauf folgenden Verhandlungen war am Rande mehrfach zu erfahren, dass man weder auf die hohe fachliche und ethische Qualität der christlichen Ausbildung verzichten wolle, noch auf das daraus hervorgehende Personal. Man habe von diesen Mitarbeiterinnen und Mitarbeitern viel zu lernen und zu übernehmen.

Katholische Kindergärten:

Wenn nichtchristliche Kinder in kirchlichen Kindergärten von Gott oder von Heiligen hörten und beten lernten, trugen sie dies in ihre Familien. Freiheit und Geborgenheit, Liebe und Zuwendung, Verlässlichkeit und politische Unabhängigkeit waren einige Gründe für die Beliebtheit der kirchlich-caritativen Angebote. Immer muss dabei auch die Diasporasituation im Blick behalten werden - die Caritas konnte nie flächendeckend präsent sein. Die vorhandenen Angebote standen allen offen. Auch nichtkatholische Kinder erlebten dankbar die ideologisch offenen kirchlichen Angebote, in denen Ver-

\footnotetext{
${ }^{5}$ Es gab kaum noch öffentliche Aktionen gegen die Kirche, wohl aber oft große Schikanen und Benachteiligungen Jugendlicher, die sich der Jugendweihe widersetzten, obwohl die Teilnahme offiziell freiwillig war. Ähnlich erging es häufig den jungen Männern, die sich dem Waffendienst dadurch entzogen, dass sie die offizielle Variante des Wehrdienstes bei den „Bausoldaten“ wählten.
} 
trauen auf die eigenen Kräfte gesetzt wurde und nicht ständig die Macht von oben erlebt wurde. In diesem Zusammenhang sind zwei gemeinsame Erfindungen von Pastoral und Caritas zu nennen:

Frohe Herrgottsstunden:

Da keine neuen katholischen Kindergärten genehmigt wurden, wurden in sehr vielen Gemeinden ein- bis dreimal pro Woche die Vorschulkinder im Pfarrhaus gesammelt. Bei Spiel und Katechese lernten sie einander kennen und wuchsen so in die Gemeinde hinein.

Religiöse Kinderwochen:

In den Schulferien fanden nach einem einheitlichen Thema gestaltete Veranstaltungen für Kinder statt. Staatliche Einwände wurden von den Bischöfen mit Verweis auf die Verfassung der DDR zurückgewiesen, denn diese erteilte der Kirche das Recht auf religiöse Unterweisung der Kinder.

\section{Professionalität und Qualität}

Die kirchlichen Ausbildungsstätten waren vom Staat völlig unabhängig. Es existierten zwei Seminare für kirchliche Erzieherinnen, ein Seminar für Sozialarbeiter, ein Seminar für Sozialpädagogen (Jugendleiter), eine Ausbildungsstätte für Altenpflegerinnen. Allerdings wurden die Abschlüsse dieser Seminare auch nicht staatlich anerkannt, sondern allein im Bereich kirchlichen Dienstes. Dies hatte aber einen ungeheuren Vorteil, weil durch mühsam aus Westdeutschland eingeschmuggelte Literatur, durch den Besuch von Professoren, Praktikern und Experten der verschiedensten Fachbereiche eine permanente Möglichkeit des Lernens und des Austauschs bestand. Diese Vorträge, Gastvorlesungen, Fort- und Weiterbildungsveranstaltungen wurden sehr sorgfältig organisiert, so dass der Staat relativ wenig davon erfuhr und sich nicht dadurch provoziert fühlte. So gelang es, in fast allen Bereichen den Anschluss an die europäischen Entwicklungen, an westliche Standards und an die Entwicklungen in der Caritas weitgehend zu behalten. Die Krankenpflegeausbildung hingegen an den kirchlichen Ausbildungskrankenhäusern wurde zwischen Kirche und Staat vertraglich geregelt und staatlich finanziert. Das Umfeld war im Unterschied zu den anderen Ostblockländern auf der fachlichen Ebene häufig sehr tolerant. Man sah und schätzte die Arbeit der Caritas. Dies wurde besonders deutlich in den Monaten des Neubeginns nach der Wende.

\section{Was kann man daraus lernen?}

Caritas kann nur gelebt werden als Teil der Kirche. Sie findet unter den verschiedensten Situationen jeweils ihre mögliche Form. Die Gemeinsamkeit von Pastoral und Caritas und die Gemeinde als Ort kirchlichen Lebens sind wichtige Erfahrungen der Katholischen Kirche in Ostdeutschland. Die geringe Kraft einer Diasporakirche erfordert entweder wirksame Hilfen von anderen Ortskirchen oder gezielte Selbstbeschränkung. Der enge Austausch mit dem Deutschen Caritasverband war Lebenskraft und 
sicherte die Qualität der Arbeit. Auch für die Caritasarbeit gilt, dass nicht die großen systemischen Strukturen, sondern die gelebte Solidarität den größten Zeugnischarakter hat.

\section{Referenzen}

Grande, D. \& J. TAMmer (1990) Diakonia: Aus Liebe dienen: Ein Meditations- und Sachbuch zum Thema Nächstenliebe (Leipzig: St. Benno).

GRÜTZ, R. (2004) Katholizismus in der DDR-Gesellschaft 1960-1990 (Paderborn: Schöningh). HöLlEN, M. (1994) Loyale Distanz? (Berlin: Martin Höllen).

JunG, R. (2003) Ungeteilt im geteilten Berlin? (Berlin: Morus).

KÖSTERS, CH., Hrsg. (2001a) Caritas in der SBZ/DDR 1945-1989 (Paderborn: Schöningh).

KÖSTERS, CH., Hrsg. (2001b) Staatssicherheit und Caritas 1959-1998 (Paderborn: Schöningh). KÖSTERS, CH. \& W. TisCHNER, Hrsg. (2005) Katholische Kirche in SBZ und DDR (Paderborn: Schöningh).

KROLL, S. (1998) Kirchlich-caritative Ausbildung in der DDR (Freiburg: Lambertus).

LANGE, G., Hrsg. (1992) Katholische Kirche-Sozialistischer Staat DDR (Leipzig: St. Benno).

LANGE, G. \& U. Pruss (1996) An der Nahtstelle der Systeme (Leipzig: Aschendorff).

PILvouseK, J. (1994) Kirchliches Leben im totalitären Staat (Leipzig: St. Benno).

SCHÄFER, B. (1998) Staat und Katholische Kirche in der DDR (Köln: Böhlau). 


\section{ANHANG}

Die katholischen sozialen Einrichtungen der Caritas
im Bereich der Berliner Bischofskonferenz

a) Einrichtungen

\begin{tabular}{|c|c|c|c|c|c|}
\hline & \multirow{2}{*}{$\begin{array}{c}\text { Einrich- } \\
\text { tungen }\end{array}$} & \multirow{2}{*}{$\begin{array}{l}\text { Plätzel } \\
\text { Betten }\end{array}$} & \multicolumn{3}{|c|}{ Hauptberufliche Mitarbeiter } \\
\hline & & & $\begin{array}{l}\text { Insge- } \\
\text { samt }\end{array}$ & $\begin{array}{c}\text { Darunter } \\
\text { Ordens- } \\
\text { gehörige }\end{array}$ & $\begin{array}{l}\text { Auf Vollzeit- } \\
\text { beschäftigte } \\
\text { umgerechnet }\end{array}$ \\
\hline Krankenhäuser & 32 & 5.065 & 6.356 & 318 & 4.765 \\
\hline Kurheime & 5 & 344 & 44 & 4 & 39 \\
\hline Erholungsheime & 24 & 676 & 160 & 37 & 98 \\
\hline Gemeindeambulanzen & 98 & - & 113 & 41 & 61 \\
\hline Säuglingsheime (0-3 J.) & 5 & 280 & 153 & 14 & 115 \\
\hline Kinderheime (ab 3. J.) & 12 & 303 & 119 & 11 & 96 \\
\hline Wohnheime f. Jgdl. & 2 & 30 & 10 & - & 10 \\
\hline Kinderkrippen & 1 & 15 & 2 & - & 2 \\
\hline Kindergärten & 140 & 7.295 & 777 & 23 & 674 \\
\hline Kinderhorte & 9 & 221 & 17 & 2 & 13 \\
\hline Alten-/Pflegeheime & 103 & 3.498 & 1.467 & 167 & 1.113 \\
\hline Heime f. Körperbehind. & 1 & 10 & 11 & - & 11 \\
\hline Heime f. geist. Behind. & 19 & 922 & 657 & 89 & 491 \\
\hline Tagesstätt.f. geist. B. & 4 & 96 & 40 & - & 36 \\
\hline Bahnhofsmission & 1 & 14 & 3 & - & 3 \\
\hline Beratungsstellen & 9 & - & 28 & - & 25 \\
\hline Caritassekretariate & 60 & - & 141 & - & 113 \\
\hline \multirow[t]{2}{*}{ Ausbildungsstätten } & 47 & 1.288 & - & - & - \\
\hline & 572 & 20.057 & 10.098 & 706 & 7.638 \\
\hline
\end{tabular}

${ }^{6}$ Caritasjahrbuch 92 (1991) (Freiburg: Deutscher Caritasverband) 336. 
b) Ausbildungsstätten und in Ausbildung befindliche Mitarbeiter

\begin{tabular}{lccc}
\hline & Ausbildungsstätten & Ausbildungsplätze & $\begin{array}{c}\text { zur Ausbildung } \\
\text { delegierte Mitarb. }\end{array}$ \\
\hline Aspiranturen & 8 & 84 & 65 \\
Praktikanturen & 22 & 239 & 185 \\
Gruppenerzieherin & 1 & 16 & 16 \\
Kindergärtnerin & 2 & 130 & 77 \\
Kinder-/Krankenpflege & 9 & 750 & 551 \\
Sozialpädagogin & 1 & 24 & 9 \\
(Jugendleit.) & 1 & 23 & 17 \\
Fürsorger/in & 2 & - & 55 \\
Altenpfleger/in & 1 & 22 & 20 \\
Koch/Köchin & 47 & 1.288 & 995 \\
\hline
\end{tabular}

Zum Vergleich: Gesamtzahlen der Caritas in der Bundesrepublik Deutschland 1990:

$\begin{array}{lr}\text { Einrichtungen: } & 30.147 \\ \text { Plätze/Betten: } & 1.073 .984 \\ \text { Vollzeitbeschäftigte: } & 250.053 \\ \text { Teilzeitbeschäftigte: } & 97.513\end{array}$

